\section{Nitrogen and Water Rates for Subsurface Trickle-irrigated Collard, Mustard, and Spinach}

\author{
Thomas L. Thompson and Thomas A. Doerge \\ Department of Soil, Water, and Environmental Science, 429 Shantz, Building \\ 38, University of Arizona, Tucson, AZ 85721
}

Additional index words. Brassica oleracea, Brassica juncea, Spinacea oleracea, soil water tension

\begin{abstract}
Three field experiments using subsurface trickle irrigation with various rates of target soil water tension (SWT) and N rates were conducted in southern Arizona during 1990-93. The experiments were conducted with collard (Brassica oleracea L. Acephela Group cv. Vates), mustard [Brassica juncea (L.) Czerniak cv. Southern Giant], and spinach (Spinacea oleracea $\mathbf{L}$. cv. Indian Summer). The interactive effects of water and $\mathbf{N}$ treatments on crop yield, $\mathbf{N}$ uptake, and unutilized fertilizer $\mathbf{N}$ were studied. In general, excessive irrigation (SWT $<5.6 \mathrm{kPa}$ ) resulted in lower yield and $\mathrm{N}$ uptake and higher unutilized fertilizer N. Optimum SWTs were $9 \mathrm{kPa}$ for collard, $8 \mathrm{kPa}$ for spinach, and 6 to $10 \mathrm{kPa}$ for mustard.
\end{abstract}

Crop production in arid regions is particularly sensitive to deficiencies of soil moisture and plant nutrients, especially N. Leafy green vegetables are often heavily fertilized with $\mathrm{N}$ and irrigated to maintain crop quality standards demanded by the market. This situation may be conducive to groundwater contamination with nitrate $\left(\mathrm{NO}_{3}^{-}\right)$. Such contamination is especially of concern in areas of the southwestern United States where production of fresh vegetables is important. For example, the percentage of wells testing above the EPA maximum contaminant level of $10 \mathrm{mg} \mathrm{NO}_{3}-\mathrm{N} /$ liter in three important vegetable production states, Arizona, California, and Texas, ranges from $9.4 \%$ to $13.9 \%$ (Fedkiw, 1991).

The use of subsurface trickle irrigation offers the possibility of maintaining crop yield, quality, and profits while reducing $\mathrm{NO}_{3}^{-}$emissions to groundwater. The greater efficiency of water application and the ability to precisely deliver needed amounts of $\mathrm{N}$ to the crop root zone account for this. Reliable information for scheduling trickle irrigation is needed, along with guidelines for plant tissue testing for N. Several methods exist for scheduling trickle irrigation, including evapotranspiration(Clough et al., 1990; Hartz, 1993; McNeish et al., 1985), allowable soil water depletion (Bogle et al., 1989), and target soil water tensions (SWTs). The advantages of using target SWTs were discussed by Pier and Doerge (1995a).

Received for publication 16 Mar. 1995. Approved for publication 11 Aug. 1995. This research was funded in part by grants from the Tennessee Valley Authority and the Arizona Dept. of Environmental Quality. The cost of publishing this paper was defrayed in part by the payment of page charges. Under postal regulations, this paper therefore must be hereby marked advertisement solely to indicate this fact.
Relatively few studies have been reported in which true "optimum" SWT values have been determined for trickle-irrigated crops from experiments with multiple levels of SWT. Phene and Beale (1976) reported that sweet corn (Zea mays L.) yields were optimized when tension was maintained at $20 \mathrm{kPa}$ at a $0.15-\mathrm{m}$ depth in a sandy loam soil. Pier and Doerge (1995a) reported an optimum SWT of $7 \mathrm{kPa}$ for trickle-irrigated watermelon [Citrullus lanatus (Thunb.) Matsum. \& Nakai] grown under hyperthermic conditions in a sandy loam soil. Feigin et al. (1982) found that subsurface trickle-irrigated celery [Apium graveolens L. var. dulce (Miller) Pers.] produced the highest yield when SWT at a 0.2-m depth was maintained at $7 \mathrm{kPa}$ in a sandy loam soil. Thompson and Doerge (1996a) found that $6 \mathrm{kPa}$ was the optimum SWT for trickleirrigated leaf lettuce (Lactuca sativa L.) grown in a sandy loam soil.

The production of leafy vegetables has recently been increasing in Arizona (Sherman and Erwin, 1993). However, to our knowledge, there is currently no information available on optimum SWTs for collard, mustard, and spinach when grown using subsurface trickle irrigation. The objectives of this study were to determine 1) the optimum SWTs for these three crops, 2) the interactive effects of water and $\mathrm{N}$ fertilizer on crop yield and net economic return, and 3) plant uptake of fertilizer $\mathrm{N}$ by the difference method as affected by $\mathrm{N}$ and water inputs.

\section{Materials and Methods}

Three field experiments using subsurface trickle irrigation were conducted at the Univ. of Arizona Maricopa Agricultural Center in southern Arizona during the 1990-93 winter growing seasons. Crops grown were commercially important cultivars of collard ('Vates'), mustard ('Southern Giant Curled'), and spinach ('Indian Summer'). The same field was used during each year. This field is mapped as a Casa Grande sandy loam [fine-loamy, mixed, hyperthermic, typic Natriargid (reclaimed)]. This soil had a $\mathrm{pH}$ of 8.5 and an organic $\mathrm{C}$ content of $0.3 \%$. The experimental area was cropped with unfertilized, flood-irrigated sudangrass (Sorghum sudanenses L.) for 5 months before winter vegetable crops were planted to reduce concentrations of available $\mathrm{N}$ in the root zone and reduce field variability. The aboveground biomass of the sudangrass was harvested and removed from the experimental area at least three times during the respective 5-month periods. Residual soil inorganic $\mathrm{N}\left(\mathrm{NH}_{4}^{+}+\mathrm{NO}_{3}\right)$ was $<4 \mathrm{mg} \cdot \mathrm{kg}^{-1}$ following the final sudangrass harvest in each year.

Trickle tubing (Twin-wall IV, 0.36-mm wall thickness, $0.23-\mathrm{m}$ emitter spacing delivering $1 \times 10^{-3}$ liters $\cdot \mathrm{s}^{-1} \cdot \mathrm{m}^{-1}$ at $70 \mathrm{kPa}$; Chapin Watermatics, Watertown, N.Y.) was buried $0.15 \mathrm{~m}$ deep directly under the midline of north-south-oriented soil beds placed $1.02 \mathrm{~m}$ apart. Six equally spaced seedlines were planted per bed using a Stanhay precision planter. Plant populations were 753,350 plants/ha (pph) for collard, $1.14 \times 10^{6} \mathrm{pph}$ for mustard, and $1.29 \times 10^{6} \mathrm{pph}$ for spinach. Uniform irrigation through the trickle tubing was continued on all plots until the stand was established (one- to two-leaf stage). Daily irrigations were then initiated by an automatic controller connected to electronic valves. Volumes of water applied by irrigation were monitored by duplicate inline propeller-type flow meters. The irrigation water used during each year had an electrical conductivity of $1.0 \mathrm{dS} \cdot \mathrm{m}^{-1}$ and an average $\mathrm{NO}_{3}-\mathrm{N}$ concentration of $2.0 \mathrm{mg} \cdot \mathrm{liter}^{-1}$.

Tensiometers, vertically inserted adjacent to the trickle tubing within one seedline, were installed in selected adequately fertilized plots of each crop within each water application treatment shortly after germination. The tensiometer cups were positioned at a depth of 0.3 $\mathrm{m}$. SWTs were measured daily before irrigation using a Tensicorder (Soil Measurement Systems, Tucson, Ariz.), as described by Marthaler et al. (1983). Irrigation was applied daily to maintain target SWT values, except when rainfall or cool weather made irrigation unnecessary. Daily changes in SWT and expected evapotranspiration for the coming day were considered when making decisions regarding daily irrigation volumes. During all three years, no pesticides were used and weed control was accomplished by hand.

All $\mathrm{N}$ fertilizer was supplied as a solution of urea ammonium-nitrate $(320 \mathrm{~g} \mathrm{~N} / \mathrm{kg}$ ). Fertilizer was injected directly into the irrigation water using venturi-type chemigators (Performance Products, Coolidge, Ariz.). All other essential plant nutrients were applied uniformly and were not limiting to plant growth. Aboveground portions of plants were harvested from sections ( 1 to $3 \mathrm{~m}^{2}$ ) within each plot when plants were at harvestable size. Fresh weight was measured, and plants were dried in a forced-air oven at $65 \mathrm{C}$, ground, and analyzed for total $\mathrm{N}$.

During the 1990-91 growing season, an N rate experiment was conducted, consisting of 
a split-plot design with three $\mathrm{N}$ rates as main plots and collard or mustard cultivars as the subplots (Table 1). A second randomized complete-block experiment with three $\mathrm{N}$ rates was conducted using spinach. The purpose of this initial experiment was to develop estimates of $\mathrm{N}$ and water requirements for these species. Plots were seeded on 25 Oct. Plots consisted of four beds, each $12.2 \mathrm{~m}$ long. Sprinkler irrigation was used for 8 days following seeding to enhance uniform germination and stand establishment. A total of $267 \mathrm{~mm}$ of water was applied during this period. Beginning on 31 Oct., sprinklers were removed and trickle irrigation was used to provide an adequate but not excessive amount of water. Irrigation water was applied in amounts necessary to maintain SWT at or near $7.0 \mathrm{kPa}$. The seasonal average SWT and irrigation water applied are shown in Table 2 . A total of $123 \mathrm{~kg} \mathrm{P} /$ ha from phosphoric acid was applied uniformly to all plots through the trickle tubing on 8 Nov. Harvest was 13 Feb. for collard and spinach. Mustard plots were harvested initially on 17 Jan. by clipping the plants at a height of 2 to $4 \mathrm{~cm}$ above ground level. Mustard was allowed to regrow and harvested again on $28 \mathrm{Feb}$. A total of $60 \mathrm{~mm}$ of rainfall fell between planting and $13 \mathrm{Feb}$., and a total of $70 \mathrm{~mm}$ between planting and 28 Feb. The seasonal potential evapotranspiration was $312 \mathrm{~mm}$ for 26 Oct.-13 Feb. and $369 \mathrm{~mm}$ for 26 Oct.-28 Feb. Potential evapotranspiration was determined by a weather station located near the experimental plots, and a modified Penman equation (Pruitt and Doorenbos, 1977).

During the 1991-92 growing season, the field experiment consisted of a $2 \times 4$ factorial randomized complete-block design with four replications. The same cultivars used in 1990 91 were planted. Four cumulative $\mathrm{N}$ rates ranging from 90 to $400 \mathrm{~kg} \cdot \mathrm{ha}^{-1}$ were applied in four or five split applications (Table 1). These rates were intended to supply $\mathrm{N}$ over the range from deficient to excessive. Target SWTs were 7.0 and $4.0 \mathrm{kPa}$. Seasonal average tensions and cumulative irrigation water totals are shown in Table 2. Plots consisted of three beds, each $12.2 \mathrm{~m}$ long. Seeds were planted on 14 Nov. A total of $843 \mathrm{~mm}$ of irrigation water was applied through the trickle tubing during the stand establishment period (14-27 Nov.), and irrigation treatments were initiated on $17 \mathrm{Dec}$. The higher amount of water needed for stand establishment in 1991-92 compared to 199091 was due to the use of trickle tubing rather than sprinklers and deeper than normal placement of the trickle tubing. Triple superphosphate $(123 \mathrm{~kg} \mathrm{P} / \mathrm{ha})$ was applied uniformly to the field and incorporated before planting. Harvest was $28 \mathrm{Feb}$. for collard and 4 Mar. for spinach. Mustard was harvested initially on 10 Feb. Mustard was allowed to regrow and final harvest was on 13 Mar. Rainfall received between planting and $28 \mathrm{Feb}$. was $111 \mathrm{~mm}$, between planting and 4 Mar. was $123 \mathrm{~mm}$, and between planting and 13 Mar. was $135 \mathrm{~mm}$. Potential evapotranspiration was $218 \mathrm{~mm}$ for 14 Nov.-28 Feb., $228 \mathrm{~mm}$ for 14 Nov.-4 Mar., and $252 \mathrm{~mm}$ for 14 Nov.-13 Mar.

During the 1992-93 growing season, the

Table 1. Schedule of $\mathrm{N}$ fertilizer applications for collard, mustard, and spinach for the 1990-93 seasons.

\begin{tabular}{|c|c|c|c|c|c|c|c|c|}
\hline \multirow[b]{2}{*}{ Year } & \multirow[b]{2}{*}{ Crop(s) } & \multirow{2}{*}{$\begin{array}{c}\text { Growth } \\
\text { stage }\end{array}$} & \multirow[b]{2}{*}{$\mathrm{DAP}^{z}$} & \multirow[b]{2}{*}{$\mathrm{DCD}^{y}$} & \multicolumn{4}{|c|}{ Nitrogen rate $\left(\mathrm{kg} \cdot \mathrm{ha}^{-1}\right)$} \\
\hline & & & & & 1 & 2 & 3 & 4 \\
\hline \multirow[t]{12}{*}{ 1990-91 } & Collard and & 1-2 Leaf & 19 & 332 & 0 & 15 & 30 & -- \\
\hline & mustard & 3-4 Leaf & 40 & 596 & 20 & 40 & 60 & --- \\
\hline & Collard and & 5-8 Leaf & 54 & 721 & 30 & 60 & 90 & --- \\
\hline & mustard & 8-10 Leaf & 77 & 824 & 20 & 40 & 60 & --- \\
\hline & Collard and & Preharvest & 105 & 1065 & 20 & 30 & 40 & --- \\
\hline & mustard & Total & & & 90 & 185 & 280 & --- \\
\hline & Spinach & 1-2 Leaf & 19 & 332 & 0 & 15 & 30 & --- \\
\hline & Spinach & 3-4 Leaf & 40 & 596 & 20 & 40 & 60 & --- \\
\hline & Spinach & 5-8 Leaf & 54 & 721 & 30 & 50 & 70 & --- \\
\hline & Spinach & 10-15 Leaf & 82 & 864 & 30 & 50 & 70 & -- \\
\hline & Spinach & Preharvest & 105 & 1065 & 0 & 20 & 40 & --- \\
\hline & Spinach & Total & & & 80 & 170 & 270 & --- \\
\hline \multirow{17}{*}{ 1991-92 } & Collard & 1-2 Leaf & 33 & 286 & 10 & 15 & 30 & 50 \\
\hline & Collard & 4 Leaf & 50 & 418 & 30 & 50 & 80 & 100 \\
\hline & Collard & 6-8 Leaf & 71 & 549 & 20 & 40 & 60 & 80 \\
\hline & Collard & 8-10 Leaf & 92 & 798 & 30 & 50 & 70 & 90 \\
\hline & Collard & Total & & & 90 & 155 & 240 & 320 \\
\hline & Mustard & 2 Leaf & 33 & 286 & 10 & 15 & 30 & 50 \\
\hline & Mustard & 4-5 Leaf & 50 & 418 & 30 & 50 & 80 & 100 \\
\hline & Mustard & 6-7 Leaf & 71 & 549 & 20 & 40 & 60 & 80 \\
\hline & Mustard & First harvest & 92 & 798 & 30 & 50 & 70 & 90 \\
\hline & Mustard & Mid-regrowth & 106 & 960 & 0 & 45 & 60 & 80 \\
\hline & Mustard & Total & & & 100 & 200 & 300 & 400 \\
\hline & Spinach & 1-2 Leaf & 33 & 286 & 0 & 20 & 30 & 50 \\
\hline & Spinach & 3-4 Leaf & 47 & 398 & 20 & 40 & 55 & 75 \\
\hline & Spinach & 6-8 Leaf & 61 & 485 & 40 & 60 & 80 & 100 \\
\hline & Spinach & 9-11 Leaf & 78 & 624 & 30 & 40 & 60 & 80 \\
\hline & Spinach & Preharvest & 92 & 798 & 10 & 20 & 45 & 55 \\
\hline & Spinach & Total & & & 100 & 180 & 270 & 360 \\
\hline \multirow[t]{12}{*}{ 1992-93 } & Collard & 1 Leaf & 32 & 296 & 0 & 20 & 40 & 40 \\
\hline & Collard & 2-3 Leaf & 51 & 424 & 20 & 30 & 60 & 90 \\
\hline & Collard & 5-6 Leaf & 74 & 644 & 40 & 60 & 80 & 150 \\
\hline & Collard & 6-7 Leaf & 87 & 789 & 20 & 40 & 70 & 100 \\
\hline & Collard & 7-9 Leaf & 105 & 977 & 0 & 20 & 30 & 50 \\
\hline & Collard & Total & & & 80 & 170 & 280 & 450 \\
\hline & Spinach & 2 Leaf & 32 & 296 & 0 & 20 & 40 & 40 \\
\hline & Spinach & 4 Leaf & 51 & 424 & 20 & 30 & 60 & 90 \\
\hline & Spinach & 7-9 Leaf & 74 & 644 & 40 & 60 & 80 & 150 \\
\hline & Spinach & 9-12 Leaf & 87 & 789 & 20 & 40 & 70 & 100 \\
\hline & Spinach & 12-14 Leaf & 105 & 977 & 0 & 20 & 30 & 50 \\
\hline & Spinach & Total & & & 80 & 170 & 280 & 450 \\
\hline
\end{tabular}

${ }^{2} \mathrm{DAP}=$ days after planting.

${ }^{y} \mathrm{DCD}$ refers to accumulated heat units between 30 and $7 \mathrm{C}$.

Table 2. Target soil water tension (SWT), actual SWT, and amounts of water applied to collard, mustard, and spinach during 1990-93.

\begin{tabular}{|c|c|c|c|c|c|}
\hline \multirow[b]{2}{*}{ Year } & \multirow[b]{2}{*}{ Crop } & \multirow{2}{*}{$\begin{array}{l}\text { Irrigation } \\
\text { treatment }\end{array}$} & \multicolumn{2}{|c|}{ SWT (kPa) } & \multirow{2}{*}{$\begin{array}{l}\text { Water } \\
\text { applied } \\
(\mathrm{mm})^{\mathrm{y}}\end{array}$} \\
\hline & & & Target & $\operatorname{Avg}^{2}$ & \\
\hline \multirow[t]{2}{*}{ 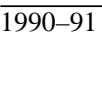 } & $\begin{array}{c}\text { Collard and } \\
\text { mustard }\end{array}$ & Optimum & 7.0 & $8.5(2.7)$ & 389 \\
\hline & Spinach & Optimum & 7.0 & $6.6(1.3)$ & 307 \\
\hline \multirow[t]{6}{*}{ 1991-92 } & Collard & Optimum & 7.0 & $6.5(1.4)$ & 246 \\
\hline & Collard & Excessive & 4.0 & $5.6(1.0)$ & 978 \\
\hline & Mustard & Optimum & 7.0 & $6.5(1.4)$ & 488 \\
\hline & Mustard & Excessive & 4.0 & $5.6(1.0)$ & 407 \\
\hline & Spinach & Optimum & 7.0 & $6.4(1.4)$ & 203 \\
\hline & Spinach & Excessive & 4.0 & $4.2(1.4)$ & 637 \\
\hline \multirow{6}{*}{ 1992-93 } & Collard & Deficient & 12.0 & $10.9(4.9)$ & 74 \\
\hline & Collard & Optimum & 7.0 & $6.6(1.1)$ & 272 \\
\hline & Collard & Excessive & 4.0 & $5.1(0.9)$ & 450 \\
\hline & Spinach & Deficient & 12.0 & $9.2(3.2)$ & 74 \\
\hline & Spinach & Optimum & 7.0 & $6.7(1.0)$ & 272 \\
\hline & Spinach & Excessive & 4.0 & $4.9(0.8)$ & 450 \\
\hline
\end{tabular}

${ }^{\mathrm{z}}$ Average of SWT measured daily before irrigation. Standard deviations are given in parentheses. 'Water applied following the stand establishment period.

field experiment consisted of a $3 \times 4$ factorial randomized complete-block design with four replications. Due to limited resources, collard and spinach only were planted. Four cumulative $\mathrm{N}$ rates of 80 to $450 \mathrm{~kg} \cdot \mathrm{ha}^{-1}$ were applied in five split applications (Table 1). The three target SWTs were 12.0, 7.0, and $4.0 \mathrm{kPa}$. Seasonal average tensions and irrigation water totals are shown in Table 2. Plots consisted of fourbeds, each $12.2 \mathrm{~m}$ long. Seeds were planted 
Soll Management, Fertilization, \& Irrigation

on 6 Nov. A total of $618 \mathrm{~mm}$ of irrigation water was applied through the trickle tubing during the stand establishment period (6 Nov.-10 Dec.), and irrigation treatments were initiated on 10 Dec. Triple superphosphate $(123 \mathrm{~kg} \mathrm{P} /$ ha) was applied uniformly to the field and incorporated before planting. Harvest was 1 Mar. for collard and 3 Mar. for spinach. Rainfall received between planting and harvest for both crops was $163 \mathrm{~mm}$. Potential evapotranspiration was $206 \mathrm{~mm}$ for 6 Nov.-1 Mar. and $212 \mathrm{~mm}$ for 6 Nov.-3 Mar. Control plots installed during this year received no $\mathrm{N}$ fertilizer and the medium irrigation treatment.

Unutilized fertilizer $\mathrm{N}\left(\mathrm{UnN}_{\mathrm{i}}\right)$ was calculated by:

$\mathrm{UnN}_{\mathrm{i}}=\mathrm{FN}_{\mathrm{i}}+\left(\mathrm{WN}_{\mathrm{i}}-\mathrm{WN}_{\mathrm{o}}\right)-\left(\mathrm{PN}_{\mathrm{i}}-\mathrm{PN}_{\mathrm{o}}\right)[\mathrm{Eq} .1]$

where $\mathrm{UnN}_{\mathrm{i}}=$ unutilized fertilizer $\mathrm{N}$ in plot $\mathrm{i}$; $\mathrm{FN}_{\mathrm{i}}=$ fertilizer $\mathrm{N}$ applied to plot $\mathrm{i} ; \mathrm{WN}_{\mathrm{i}}=\mathrm{N}$ applied in irrigation water to plot $\mathrm{i} ; \mathrm{WN}_{\mathrm{o}}=\mathrm{N}$ applied in irrigation water to control plot; $\mathrm{PN}_{\mathrm{i}}$ $=$ total crop $\mathrm{N}$ uptake in plot $\mathrm{i}$; and $\mathrm{PN}_{\mathrm{o}}=$ total crop $\mathrm{N}$ uptake in control plot harvest areas receiving no $\mathrm{N}$ fertilizer. Plant $\mathrm{N}$ in the control plots $\left(\mathrm{PN}_{\mathrm{o}}\right)$ was not available before 1992-93. Therefore, plant $\mathrm{N}$ uptake was not adjusted for $\mathrm{PN}_{\mathrm{o}}$ during 1990-91 and 1991-92. However, only $7 \mathrm{~kg} \mathrm{~N} / \mathrm{ha}$ were taken up by plants in control plots during 1992-93. Therefore, failure to correct for $\mathrm{PN}_{\mathrm{o}}$ probably introduced negligible error and did not materially affect the results. $\mathrm{PN}_{\mathrm{o}}$ represents crop $\mathrm{N}$ uptake from this field following exhaustive cropping. Any differences in $\mathrm{N}$ uptake between fertilized and control plots were assumed to be the result of the $\mathrm{N}$ and water treatments, or their effects on plant growth and $\mathrm{N}$ recovery in plant biomass.

Regression models were fitted to data for yield, $\mathrm{N}$ uptake, and unutilized fertilizer $\mathrm{N}$ (SAS Institute, 1988). For the 1992-93 data, response surfaces for yield and unutilized fertilizer $\mathrm{N}$ were determined using the SAS statistical procedure PROC RSREG. Response surfaces were used only during 1992-93 because this was the only year with more than two levels each of water and $\mathrm{N}$.

\section{Results and Discussion}

Collard. Collard yields during 1990-91 (Table 3) increased with each increasing increment of $\mathrm{N}$ fertilizer. Therefore, excessive rates of $\mathrm{N}$ were apparently not achieved. Nitrogen uptake and unutilized fertilizer $\mathrm{N}$ also increased with increasing $\mathrm{N}$ rate. Unutilized $\mathrm{N}$ includes $\mathrm{N}$ remaining in the soil, and $\mathrm{N}$ lost by various processes, including leaching of $\mathrm{NO}_{3}^{-}$. During 1991-92, maximum observed collard yields were similar to those in 1990-91. At equivalent $\mathrm{N}$ rates, yield and $\mathrm{N}$ uptake were lower, and unutilized fertilizer $\mathrm{N}$ was higher at $5.6 \mathrm{kPa}$ compared to $6.5 \mathrm{kPa}$. At $5.6 \mathrm{kPa}$, irrigation apparently was excessive. Application of the higher amount of water significantly reduced collard yield even at the highest $\mathrm{N}$ rate $(320 \mathrm{~kg} \mathrm{~N} / \mathrm{ha})$. Especially high values for unutilized fertilizer $\mathrm{N}$ were noted at 5.6 $\mathrm{kPa}$. There were, in general, significant interactions between $\mathrm{N}$ rate and SWT. During

Table 3. Yield, N uptake, and unutilized N for collard 1990-93.

\begin{tabular}{|c|c|c|c|c|c|}
\hline Year & $\begin{array}{c}\text { Soil water } \\
\text { tension } \\
(\mathrm{kPa})\end{array}$ & $\begin{array}{c}\mathrm{N} \\
\left(\mathrm{kg}^{-} \mathrm{ha}^{-1}\right)\end{array}$ & $\begin{array}{c}\text { Yield } \\
\left(\mathrm{Mg} \cdot h \mathrm{a}^{-1}\right)\end{array}$ & $\begin{array}{c}\mathrm{N} \\
\text { uptake } \\
\left(\mathrm{kg}^{2} \mathrm{ha}^{-1}\right)\end{array}$ & $\begin{array}{c}\text { Unutilized } \\
\mathrm{N}^{2} \\
\left(\mathrm{~kg}^{2} \mathrm{ha}^{-1}\right)\end{array}$ \\
\hline \multirow[t]{3}{*}{$\overline{1990-91}$} & 8.5 & 90 & 18.3 & 79 & 17 \\
\hline & 8.5 & 185 & 30.9 & 161 & 40 \\
\hline & 8.5 & 280 & 40.4 & 205 & 91 \\
\hline \multicolumn{6}{|l|}{ Significance } \\
\hline Replicatio & & & NS & NS & NS \\
\hline$N-L^{y}$ & & & $* * *$ & $* * *$ & $* * *$ \\
\hline $\mathrm{N}-\mathrm{Q}$ & & & NS & $*$ & $*$ \\
\hline$R^{2}$ & & & 0.96 & 0.97 & 0.94 \\
\hline \multirow[t]{8}{*}{ 1991-92 } & 6.5 & 90 & 24.6 & 77 & 25 \\
\hline & 6.5 & 155 & 37.4 & 100 & 67 \\
\hline & 6.5 & 240 & 42.4 & 160 & 93 \\
\hline & 6.5 & 320 & 47.0 & 168 & 165 \\
\hline & 5.6 & 90 & 21.2 & 64 & 53 \\
\hline & 5.6 & 155 & 32.1 & 77 & 105 \\
\hline & 5.6 & 240 & 37.1 & 113 & 154 \\
\hline & 5.6 & 320 & 41.8 & 124 & 223 \\
\hline \multicolumn{6}{|l|}{ Significance } \\
\hline Rep & & & NS & NS & NS \\
\hline $\mathrm{N}-\mathrm{L}$ & & & $* * *$ & $* * *$ & $* * *$ \\
\hline $\mathrm{N}-\mathrm{Q}$ & & & $* * *$ & $* *$ & $* *$ \\
\hline $\mathrm{N}-\mathrm{C}$ & & & * & $* * *$ & $* * *$ \\
\hline Water (W & & & $* * *$ & $* * *$ & $* * *$ \\
\hline $\mathrm{N} \times \mathrm{W}$ & & & NS & $* * *$ & $* * *$ \\
\hline $\mathrm{N}-\mathrm{Q} \times \mathrm{W}$ & & & NS & $* *$ & NS \\
\hline $\mathrm{N}-\mathrm{C} \times \mathrm{W}$ & & & NS & $* * *$ & NS \\
\hline$R^{2}$ & & & 0.93 & 0.99 & 0.99 \\
\hline \multirow[t]{12}{*}{ 1992-93 } & 10.9 & 80 & 16.9 & 50 & 32 \\
\hline & 10.9 & 170 & 32.7 & 118 & 55 \\
\hline & 10.9 & 280 & 43.8 & 180 & 103 \\
\hline & 10.9 & 450 & 47.9 & 225 & 228 \\
\hline & 6.6 & 80 & 14.3 & 46 & 40 \\
\hline & 6.6 & 170 & 31.0 & 102 & 75 \\
\hline & 6.6 & 280 & 45.2 & 162 & 125 \\
\hline & 6.6 & 450 & 50.0 & 223 & 234 \\
\hline & 5.1 & 80 & 15.9 & 48 & 42 \\
\hline & 5.1 & 170 & 31.7 & 110 & 70 \\
\hline & 5.1 & 280 & 43.3 & 151 & 139 \\
\hline & 5.1 & 450 & 45.0 & 168 & 292 \\
\hline \multicolumn{6}{|l|}{ Significance } \\
\hline Rep & & & NS & NS & NS \\
\hline $\mathrm{N}-\mathrm{L}$ & & & $* * *$ & $* * *$ & $* * *$ \\
\hline $\mathrm{N}-\mathrm{Q}$ & & & $* * *$ & $* * *$ & $* * *$ \\
\hline $\mathrm{N}-\mathrm{C}$ & & & NS & NS & NS \\
\hline W-L & & & NS & $* * *$ & $* * *$ \\
\hline W-Q & & & NS & NS & NS \\
\hline $\mathrm{N} \times \mathrm{W}$ & & & NS & ** & $* *$ \\
\hline $\mathrm{N}-\mathrm{Q} \times \mathrm{W}$ & & & NS & NS & NS \\
\hline $\mathrm{N} \times \mathrm{W}-\mathrm{Q}$ & & & $*$ & ** & $* *$ \\
\hline$R^{2}$ & & & 0.93 & 0.91 & 0.95 \\
\hline
\end{tabular}

${ }^{2}$ Only the 1992-93 data for unutilized $\mathrm{N}$ were corrected for plant $\mathrm{N}$ in the control plots $\left(\mathrm{PN}_{\mathrm{o}}\right)$. ${ }^{\mathrm{y}} \mathrm{L}=$ linear, $\mathrm{Q}=$ quadratic, $\mathrm{C}=$ cubic

ss, *, *****Nonsignificant or significant at $P \leq 0.10,0.05$, and 0.01 , respectively.

1992-93, $\mathrm{N}$ rates in excess of $280 \mathrm{~kg} \cdot \mathrm{ha}^{-1}$ resulted in only small increases in yields for each water level. Collard yield and $\mathrm{N}$ uptake generally were less responsive to irrigation during 1992-93 than during 1991-92, possibly due to the higher than average rainfall received during 1992-93. Although rainfall was above average during both years, it was distributed throughout the growing season during 1992-93, while it occurred largely just before harvest during 1991-92. Therefore, the impacts of the rainfall on plant response were more important during 1992-93 than during 1991-92. There was a trend toward lower yields at 10.9 and $5.1 \mathrm{kPa}$. The excessive $\mathrm{N}$ rate $\left(450 \mathrm{~kg} \cdot \mathrm{ha}^{-1}\right)$ resulted in marked increases in unutilized fertilizer $\mathrm{N}$, especially at $5.1 \mathrm{kPa}$. It may be expected that significant amounts of the unutilized fertilizer $\mathrm{N}$ at $5.1 \mathrm{kPa}$ did not remain in the soil, but were lost by leaching, denitrification, or both. Such a trend was reported by Thompson and Doerge (1996b) for trickle-irrigated leaf lettuce.

Mustard. Mustard yields during 1990-91 (Table 4) were maximized at the highest $\mathrm{N}$ rate; therefore, excessive $\mathrm{N}$ rates may not have been achieved. This possibility is further suggested by the very low values for unutilized fertilizer $\mathrm{N}$ measured during that season. Similarly, during 1991-92, excessive $\mathrm{N}$ rates may not have been achieved. Yields increased with increasing $\mathrm{N}$ applications up to the highest rate $\left(400 \mathrm{~kg} \cdot \mathrm{ha}^{-1}\right)$. However, yields during 199192 were lower for all $\mathrm{N}$ rates at $5.6 \mathrm{kPa}$ than for the same $\mathrm{N}$ rates at $6.5 \mathrm{kPa}$. Also, $\mathrm{N}$ uptake was lower and the quantity of unutilized fertilizer 
Table 4. Yield, N uptake, and unutilized N for mustard 1990-92.

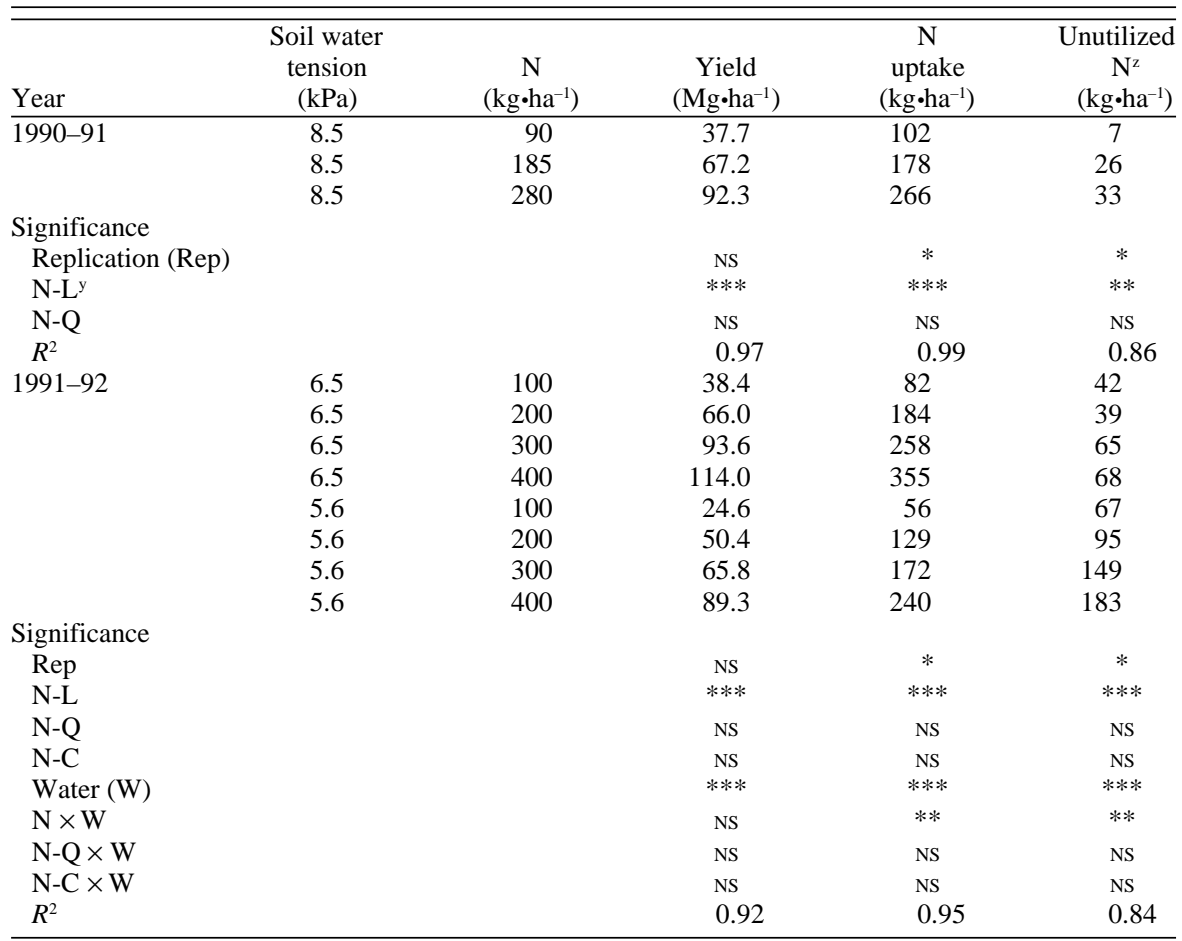

${ }^{2}$ These data were not corrected for plant $\mathrm{N}$ in the control plots $\left(\mathrm{PN}_{\mathrm{o}}\right)$.

${ }^{\mathrm{y}} \mathrm{L}=$ linear, $\mathrm{Q}=$ quadratic, $\mathrm{C}=$ cubic.

Ns, ${ }^{*}, * *, * * *$ Nonsignificant or significant at $P \leq 0.10,0.05$, and 0.01 , respectively.

Table 5. Yield, $\mathrm{N}$ uptake, and unutilized $\mathrm{N}$ for spinach 1990-93.

\begin{tabular}{|c|c|c|c|c|c|}
\hline Year & $\begin{array}{c}\text { Soil water } \\
\text { tension } \\
(\mathrm{kPa})\end{array}$ & $\begin{array}{c}\mathrm{N} \\
\left(\mathrm{kg} \cdot \mathrm{ha}^{-1}\right)\end{array}$ & $\begin{array}{c}\text { Yield } \\
\left(\mathrm{Mg} \cdot h a^{-1}\right)\end{array}$ & $\begin{array}{c}\mathrm{N} \\
\text { uptake } \\
\left(\mathrm{kg}^{2} \cdot \mathrm{ha}^{-1}\right)\end{array}$ & $\begin{array}{c}\text { Unutilized } \\
\mathrm{N}^{\mathrm{z}} \\
\left(\mathrm{kg} \cdot \mathrm{ha}^{-1}\right)\end{array}$ \\
\hline \multirow[t]{3}{*}{ 1990-91 } & 6.6 & 80 & 8.6 & 72 & 23 \\
\hline & 6.6 & 175 & 16.1 & 134 & 55 \\
\hline & 6.6 & 270 & 23.2 & 190 & 95 \\
\hline \multicolumn{6}{|c|}{ Significance } \\
\hline Replicati & & & NS & NS & NS \\
\hline $\mathrm{N}-\mathrm{L}^{y}$ & & & $* * *$ & $* * *$ & $* * *$ \\
\hline $\mathrm{N}-\mathrm{Q}$ & & & NS & NS & NS \\
\hline$R^{2}$ & & & 0.97 & 0.97 & 0.93 \\
\hline \multirow[t]{8}{*}{ 1991-92 } & 6.4 & 100 & 28.4 & 124 & -11 \\
\hline & 6.4 & 180 & 42.2 & 178 & 15 \\
\hline & 6.4 & 270 & 44.5 & 173 & 109 \\
\hline & 6.4 & 360 & 45.0 & 190 & 183 \\
\hline & 4.2 & 100 & 23.9 & 101 & 21 \\
\hline & 4.2 & 180 & 29.7 & 132 & 69 \\
\hline & 4.2 & 270 & 38.7 & 153 & 139 \\
\hline & 4.2 & 360 & 35.3 & 139 & 242 \\
\hline \multicolumn{6}{|c|}{ Significance } \\
\hline Rep & & & NS & NS & NS \\
\hline $\mathrm{N}-\mathrm{L}$ & & & $* * *$ & $* * *$ & $* * *$ \\
\hline $\mathrm{N}-\mathrm{Q}$ & & & $* * *$ & $* * *$ & $* * *$ \\
\hline $\mathrm{N}-\mathrm{C}$ & & & NS & NS & NS \\
\hline Water (W & & & $* * *$ & $* * *$ & $* * *$ \\
\hline $\mathrm{N} \times \mathrm{W}$ & & & NS & NS & NS \\
\hline $\mathrm{N}-\mathrm{Q} \times \mathrm{W}$ & & & NS & NS & NS \\
\hline $\mathrm{N}-\mathrm{C} \times \mathrm{W}$ & & & $*$ & NS & NS \\
\hline$R^{2}$ & & & 0.89 & 0.83 & 0.98 \\
\hline \multirow{12}{*}{ 1992-93 } & 9.2 & 80 & 12.8 & 57 & 26 \\
\hline & 9.2 & 170 & 23.4 & 117 & 55 \\
\hline & 9.2 & 280 & 31.9 & 159 & 124 \\
\hline & 9.2 & 450 & 31.8 & 175 & 278 \\
\hline & 6.7 & 80 & 12.5 & 56 & 31 \\
\hline & 6.7 & 170 & 21.3 & 98 & 78 \\
\hline & 6.7 & 280 & 31.7 & 149 & 137 \\
\hline & 6.7 & 450 & 33.7 & 169 & 287 \\
\hline & 4.9 & 80 & 11.9 & 46 & 44 \\
\hline & 4.9 & 170 & 19.8 & 94 & 86 \\
\hline & 4.9 & 280 & 26.8 & 125 & 165 \\
\hline & 4.9 & 450 & 30.5 & 151 & 309 \\
\hline
\end{tabular}

$\mathrm{N}$ was higher at $5.6 \mathrm{kPa}$. Similar to the collard results, this result suggests that $5.6 \mathrm{kPa}$ represented excessive irrigation. Again, the application of more water decreased mustard yield, even when the highest amount of $\mathrm{N}$ was applied. Nitrogen $\times$ waterinteractions were noted for $\mathrm{N}$ uptake and unutilized fertilizer $\mathrm{N}$, but not for yield. These results illustrate the very high $\mathrm{N}$ uptake potential of subsurface trickleirrigated mustard (up to $355 \mathrm{~kg} \mathrm{~N} / \mathrm{ha}$ ) when it is supplied with adequate but not excessive irrigation. The very high values for yield and $\mathrm{N}$ uptake are no doubt due in part to the practice of multiple mustard harvests.

Spinach. Spinach yield during 1990-91 (Table 5) was maximized at the highest $\mathrm{N}$ rate; therefore, excessive $\mathrm{N}$ rates may not have been achieved. Yields for spinach during 1990-91 were lower than for the other two years because the crop was harvested at an early growth stage, due to plant disease. During 1991-92, only slight increases in yield resulted from $\mathrm{N}$ applications in excess of $180 \mathrm{~kg} \cdot \mathrm{ha}^{-1}$ at $6.4 \mathrm{kPa}$ and $270 \mathrm{~kg} \cdot \mathrm{ha}^{-1}$ at $4.2 \mathrm{kPa}$. Trends in yield and $\mathrm{N}$ uptake indicate that $4.2 \mathrm{kPa}$ represented excessive irrigation. Values for unutilized fertilizer $\mathrm{N}$ were significantly higher at $4.2 \mathrm{kPa}$. In general, the interaction terms for the various characteristics were nonsignificant. During 1992-93, yields for all irrigation treatments increased only slightly with $\mathrm{N}$ rates in excess of $280 \mathrm{~kg} \cdot \mathrm{ha}^{-1}$. There was more unutilized fertilizer $\mathrm{N}$ at $4.9 \mathrm{kPa}$ than in the other irrigation treatments. Increases in $\mathrm{N}$ rate from 280 to $450 \mathrm{~kg} \cdot h \mathrm{a}^{-1}$ resulted in only small increases in $\mathrm{N}$ uptake, but large increases in unutilized fertilizer N. As with collard and mustard, spinach yield was lower in 1991-92 and 199293 when excessive irrigation water was applied, even when an excessive $\mathrm{N}$ rate was provided.

Predicted responses. Response surfaces for predicted aboveground plant $\mathrm{N}$ uptake for collard and spinach during 1992-93 show clearly the greater $\mathrm{N}$ uptake exhibited by collard as compared to spinach (Fig. 1). Nitrogen uptake was, in general, more responsive to $\mathrm{N}$ rate than to SWT. However, at high $\mathrm{N}$ rates the $\mathrm{N}$ rate $\times \mathrm{SWT}$ interaction can be clearly seen. Nitrogen uptake at high (excessive) $\mathrm{N}$ rates was less under low SWT for both crops.

The predicted collard yield during 1992 93 was maximized at $9 \mathrm{kPa}$ and $396 \mathrm{~kg} \mathrm{~N} / \mathrm{ha}$ (Fig. 2A). Therefore, using a yield criterion, 9 kPa represents an "optimum" SWT for trickleirrigated collard. However, the 396-kg N/ha rate would have led to high levels of unutilized fertilizer N (Fig. 2B). The shaded area in Fig. $2 \mathrm{~A}$ represents $>95 \%$ of maximum predicted yield. We assumed that a response $<95 \%$ of the maximum would be unacceptable to growers. Therefore, maintaining SWT at $9 \mathrm{kPa}$ with $300 \mathrm{~kg}$ of applied N/ha would have resulted in $95 \%$ of maximum predicted yield and economic return (data not shown) with $<150 \mathrm{~kg}$ of unutilized fertilizer N/ha (Fig. 2B). Under conditions of optimum irrigation, much of this unutilized fertilizer $\mathrm{N}$ will probably remain within the crop root zone for recovery by subsequent crops (Pier and Doerge, 1995b; Thompson and Doerge, 1996b). Higher levels 
Table 5. Continued.

\begin{tabular}{|c|c|c|c|c|c|}
\hline Year & $\begin{array}{c}\text { Soil water } \\
\text { tension } \\
(\mathrm{kPa})\end{array}$ & $\begin{array}{c}\mathrm{N} \\
\left(\mathrm{kg} \cdot \mathrm{ha}^{-1}\right)\end{array}$ & $\begin{array}{c}\text { Yield } \\
\left(\mathrm{Mg}^{\prime} \mathrm{ha}^{-1}\right)\end{array}$ & $\begin{array}{c}\mathrm{N} \\
\text { uptake } \\
\left(\mathrm{kg} \cdot \mathrm{ha}^{-1}\right)\end{array}$ & $\begin{array}{c}\text { Unutilized } \\
\mathrm{N}^{\mathrm{z}} \\
\left(\mathrm{kg} \cdot \mathrm{ha}^{-1}\right)\end{array}$ \\
\hline \multicolumn{6}{|l|}{ Significance } \\
\hline Rep & & & $* *$ & NS & NS \\
\hline N-L & & & $* * *$ & $* * *$ & $* * *$ \\
\hline N-Q & & & $* * *$ & $* * *$ & **** \\
\hline $\mathrm{N}-\mathrm{C}$ & & & NS & NS & NS \\
\hline W-L & & & $* * *$ & $* * *$ & $* * *$ \\
\hline W-Q & & & $* *$ & NS & NS \\
\hline $\mathrm{N} \times \mathrm{W}$ & & & NS & NS & NS \\
\hline $\mathrm{N}-\mathrm{Q} \times \mathrm{W}$ & & & $* *$ & $*$ & $*$ \\
\hline $\mathrm{N} \times \mathrm{W}-\mathrm{Q}$ & & & $*$ & NS & NS \\
\hline$R^{2}$ & & & 0.96 & 0.95 & 0.99 \\
\hline
\end{tabular}

${ }^{\mathrm{z}}$ Only the 1992-93 data for unutilized $\mathrm{N}$ were corrected for plant $\mathrm{N}$ in the control plots $\left(\mathrm{PN}_{\mathrm{o}}\right)$. ${ }^{\mathrm{y}} \mathrm{L}=$ linear, $\mathrm{Q}=$ quadratic, $\mathrm{C}=$ cubic

Ns, ${ }^{* * *}, * * *$ Nonsignificant or significant at $P \leq 0.10,0.05$, and 0.01 , respectively.
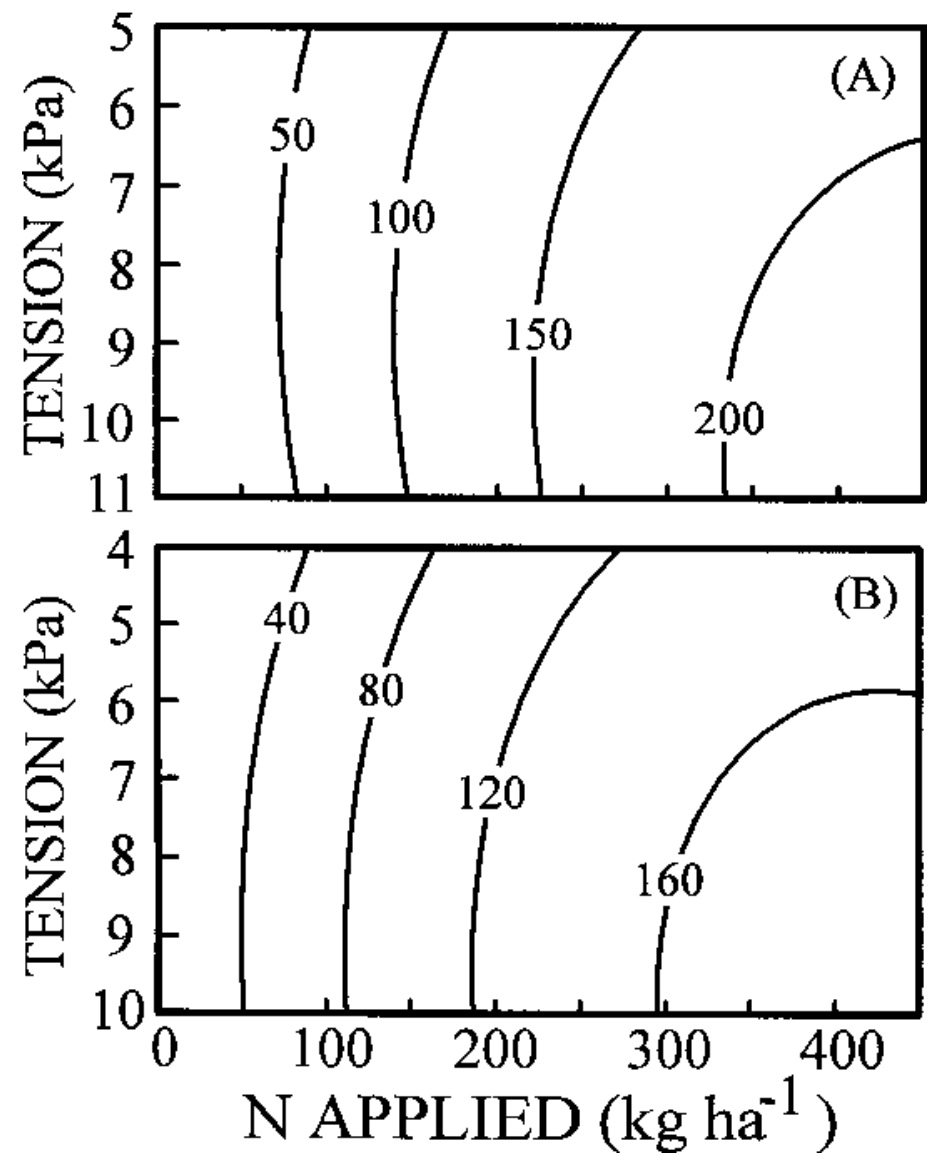

Fig. 1. (A) Predicted aboveground plant biomass $\mathrm{N}\left(\mathrm{kg} \cdot \mathrm{ha}^{-1}\right)$ for 1992-93 collard. No maximum was predicted within the range of the treatments. Model $R^{2}=0.88$, lack of fit: $P<0.378$. (B) Predicted

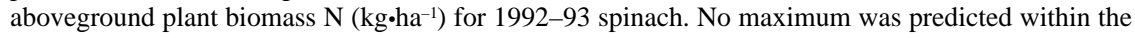
range of the treatments. Model $R^{2}=0.95$, lack of fit: $P<0.386$.

of unutilized fertilizer $\mathrm{N}$ will result if tension is maintained at less than optimum (Fig. 2B).

Because mustard was not grown with more than two irrigation treatments, it was not possible to definitively identify an optimum SWT for this species. However, results during 199192 (Table 4) demonstrated that a tension of 5.6 $\mathrm{kPa}$ represented excessive irrigation. Smittle et al. (1992) found that mustard responded best when tension did not exceed $25 \mathrm{kPa}$. However, this was the lowest tension used in their experiment. Tension likely should be maintained between 6 to $10 \mathrm{kPa}$ for optimum yields of trickle-irrigated mustard. However, additional research will be required to precisely establish an appropriate value for mustard.

Predicted spinach yields for 1992-93 (Fig. 2C) were maximized at a SWT of $8 \mathrm{kPa}$ and an $\mathrm{N}$ rate of $399 \mathrm{~kg} \cdot \mathrm{ha}^{-1}$. The high $\mathrm{N}$ rates associated with predicted maximum yields for collard and spinach were due in part to the inherently low level of organic matter in this soil and to the exhaustive cropping before each season that further contributed to very low available soil $\mathrm{N}$. This practice may not have represented normal commercial production conditions. Instead, these "optimum" $\mathrm{N}$ rates probably constitute an upper limit to the amount of fertilizer $\mathrm{N}$ needed for maximum yield of these crops. Irrigation of spinach according to a target tension of $8 \mathrm{kPa}$ and $300 \mathrm{~kg} \mathrm{~N} / \mathrm{ha}$ would have resulted in $>95 \%$ of maximum predicted yield and $<150 \mathrm{~kg}$ of unutilized fertilizer N/ha (Fig. 2D). Similar to collard, higher amounts of unutilized fertilizer $\mathrm{N}$ would have resulted with lower tensions and higher $\mathrm{N}$ rates. However, excessive $\mathrm{N}$ rates had no adverse effects on growth or quality of any of the three cultivars studied.

Three field experiments were conducted under subsurface trickle irrigation with collard, mustard, and spinach under very high yielding conditions. Yield and $\mathrm{N}$ uptake were both highly responsive to $\mathrm{N}$ applications, and less responsive to differences in SWT. In general, excessive irrigation ( $\mathrm{SWT}<5.6 \mathrm{kPa}$ ) resulted in lower yield, $\mathrm{N}$ uptake, and higher unutilized fertilizer N. Reductions in crop yield under these circumstances did not appear to result solely from reduced availability of $\mathrm{N}$ caused by leaching or denitrification. In these experiments, excessive irrigation was consistently more detrimental than deficit irrigation. Optimum SWTs identified during 1992-93 (tensions associated with maximum yield) were $9 \mathrm{kPa}$ for collard, $8 \mathrm{kPa}$ for spinach, and 6 to 10 $\mathrm{kPa}$ for mustard. Use of tensiometers to maintain SWT near these values and applications of adequate but not excessive amounts of $\mathrm{N}$ would have resulted in $>95 \%$ of predicted maximum yield and moderate levels of unutilized fertilizer $\mathrm{N}$ for collard, mustard, and spinach.

\section{Literature Cited}

Bogle, C.R., T.K. Hartz, and C. Nunez. 1989. Comparison of subsurface trickle and furrow irrigation on plastic-mulched and bare soil for tomato production. J. Amer. Soc. Hort. Sci. 114:40-43.

Clough, G.H., S.J. Locascio, and S.M. Olson. 1990. Yield of successively cropped polyethylenemulched vegetables as affected by irrigation method and fertilization management. J. Amer. Soc. Hort. Sci. 115:884-887.

Fedkiw, J. 1991. Nitrate occurrence in U.S. waters (and related questions). USDA Working Group on Water Quality. U.S. Dept. of Agr., Washington, D.C.

Feigin, A., J. Letey, and W.M. Jarrell. 1982. Celery response to type, amount and method of $\mathrm{N}$ fertilizer application under drip irrigation. Agron. J. 74:971-977.

Hartz, T.K. 1993. Drip irrigation scheduling for fresh-market tomato production. HortScience 28:35-37.

Marthaler, H.P., W. Vogelsanger, F. Richard, and P.J. Wierenga. 1983. A pressure transducer for field tensiometers. Soil Sci. Soc. Amer. J.47:624627.

McNiesh, C.M., N.C. Welch, and R.D. Nelson. 1985. Trickle irrigation requirements for strawberries in coastal California. J. Amer. Soc. Hort. Sci. 110:714-718.

Phene, C.J. and O.W. Beale. 1976. High-frequency irrigation for water and nutrient management in humid regions. Soil Sci. Soc. Amer. J. 40:430436.

Pier, J.W. and T.A. Doerge. 1995a. Nitrogen and water interactions in trickle irrigated watermelon. Soil Sci. Soc. Amer. J. 59:145-150.

Pier, J.W. and T.A. Doerge. 1995b. Concurrent evaluation of agronomic, economic, and envi- 

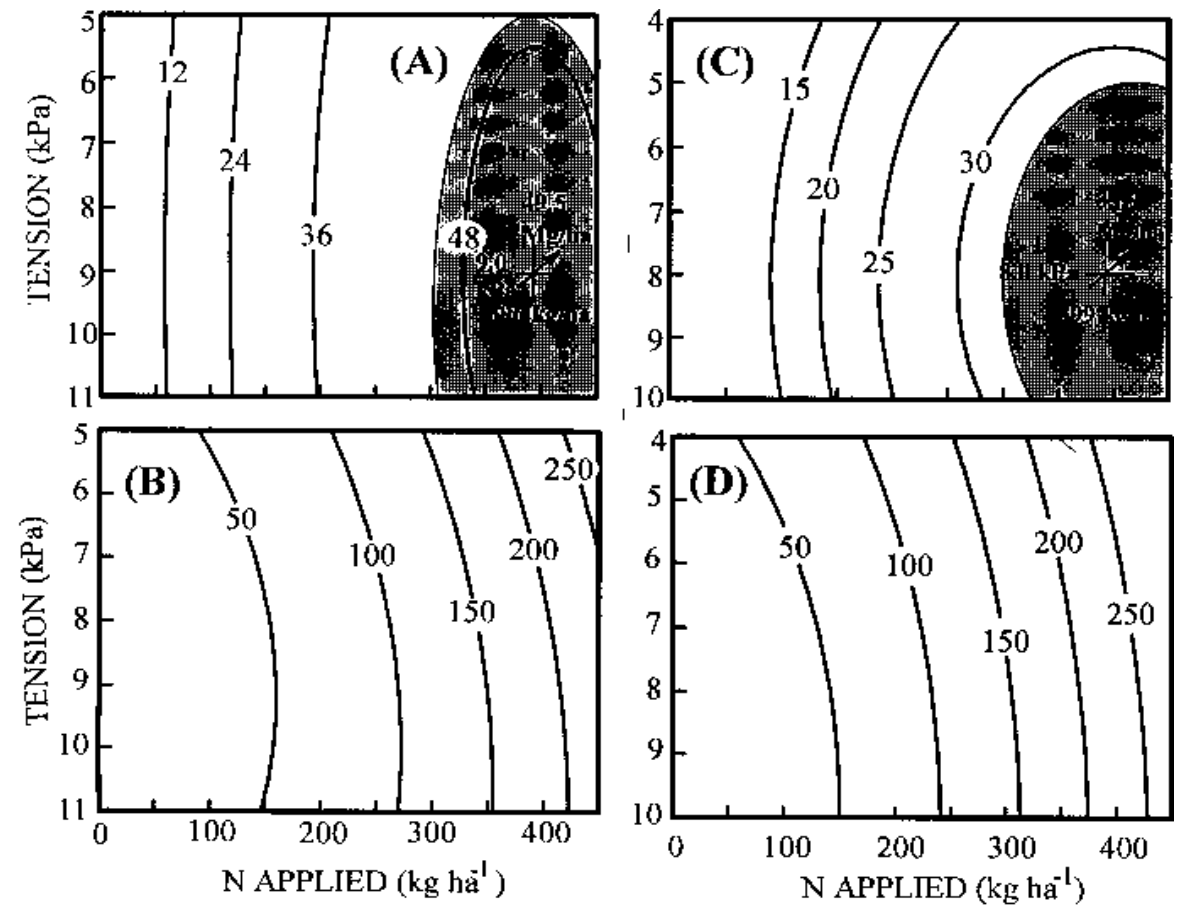

ronmental aspects of trickle irrigated watermelon production. J. Environ. Qual. 24:79-86.

Pruitt, W.O. and J. Doorenbos. 1977. Empirical calibration: A requisite for evapotranspiration formulae based on daily or longer mean climatic data. Proc. Intl. Round Table Conference on Evapotranspiration, Budapest, Hungary.

SAS Institute. 1988. SAS/STAT users guide, release 6.03 edition. SAS Inst., Cary, N.C.

Sherman, W. and B. Erwin. 1993. 1992 Arizona agricultural statistics. Bul. S-28. Arizona Agr. Sta. Serv., Phoenix.

Smittle, D.A., W.L. Dickens, J.R. Stansell, and E. Simonne. 1992. Irrigation regimes affect leaf yield and water use by turnip and mustard. HortScience 27:308-310.

Thompson, T.L. and T.A. Doerge. 1996a. Nitrogen and water interactions in subsurface trickleirrigated leaf lettuce. I. Response to water and nitrogen inputs. Soil Sci. Soc. Amer. J. (In press.)

Thompson, T.L. and T.A. Doerge. 1996b. Nitrogen and water interactions in subsurface trickleirrigated leaf lettuce. II. Evaluation of agronomic, economic, and environmental outcomes. Soil Sci. Soc. Amer. J. (In press.)

F̈ig $\overline{2}$. (̇̈) Predicted fresh weight yield $\left(\mathrm{Mg} \cdot \mathrm{ha}^{-1}\right)$ for $1992-93$ collard. Shaded area represents $>95 \%$ of maximum predicted yield. The crosshair indicates the point and the value of the maximum predicted yield. Model $R^{2}=0.89$, lack of fit: $P<0.86$. (B) Predicted unutilized fertilizer N $\left(\mathrm{kg}^{2} \mathrm{ha}^{-1}\right)$ for $1992-93$ collard. Model $R^{2}=0.93$, lack of fit: $P<0.388$. (C) Predicted fresh weight yield $\left(\mathrm{Mg} \cdot \mathrm{ha}^{-1}\right)$ for $1992-93$ spinach. Shaded area represents $>95 \%$ of maximum predicted yield. The crosshair indicates the point and the value of the maximum predicted yield. Model $R^{2}=0.93$, lack of fit: $P<0.164$. (D) Predicted unutilized fertilizer $\mathrm{N}\left(\mathrm{kg} \cdot \mathrm{ha}^{-1}\right)$ for $1992-93$ spinach. Model $R^{2}=0.99$, lack of fit: $P<0.386$. 